\section{MS10-O2 Proteopedia - a scientific 'Wiki' bridging the rift between 3D structure and function of biomacromolecules}

\author{
Joel L. Sussman ${ }^{1}$, Jaime Prilusky ${ }^{1}$
}

1. Weizmann Institute of Science

email: Joel.Sussman@weizmann.ac.il

Scientists are now able to access 3D images of biomacromolecules underlying biological functions and disease. Rather than relying on text \& $2 \mathrm{D}$ images to try to understand the function of biomacromolecular structures, a collaborative website called Proteopedia ${ }^{1,2}$ provides a new resource by linking written information \& 3D structural information. This wiki web resource, http://proteopedia.org, displays protein structures \& other biomacromolecules in an interactive format. The interactive images are surrounded by descriptive text containing hyperlinks that change the appearance (such as view, representations, colors or labels) of the adjacent 3D structure to reflect the concept discussed in the text. This makes the complex structural information readily accessible and comprehensible, even to non-structural biologists. Using Proteopedia, scientists \& students can easily create descriptions of biomacromolecules linked to their 3D structure, e.g., a page on ribosome structure/function, http://proteopedia.org/w/Ribosome. Pages can be viewed on PCs, MACs, LINUX computers $\&$ even on iPads (that do not have JAVA), via the molecular viewer $\mathrm{JSmol}^{3}$, e.g., a page on HIV-1 protease, http://proteopedia.org/w/HIV-1_protease. Content is being added by Proteopedia's $\sim 2,900$ users (in 60 different countries), in a dozen different languages, including Russian, Arabic \& Chinese: http://proteopedia.org/w/1vot_(Chinese). A number of journals \& book publishers are using Proteopedia to complement their printed and web papers using Proteopedia's "Interactive 3D Complements" (I3DCs) see, e.g.,

http://www.proteopedia.org/w/Journal:JBIC:6. Pages for each of the $>107,000$ entries in the PDB have been automatically created with 'seed' information, and are both intrinsically useful and 'primed' for expansion by users. Scientists \& students are invited to request a Proteopedia user account, at no cost, in order to edit existing pages \& to create new ones, see: http://proteopedia.org/w/Special:RequestAccount.

\section{References:}

1. Hodis, E., Prilusky, J., Martz, E., Silman, I., Moult, J. \& Sussman, J. L. (2008). Genome Biol 9, R121.

2. Prilusky, J., Hodis, E., Canner, D., Decatur, W. A., Oberholser, K., Martz, E., Berchanski, A., Harel, M. \& Sussman, J. L. (2011). J Struct Biol 175, 244.

3. Hanson, R. M., Prilusky, J., Renjian, Z., Nakane, T. \& Sussman, J. L. (2013). Israel J Chem 53, 207.

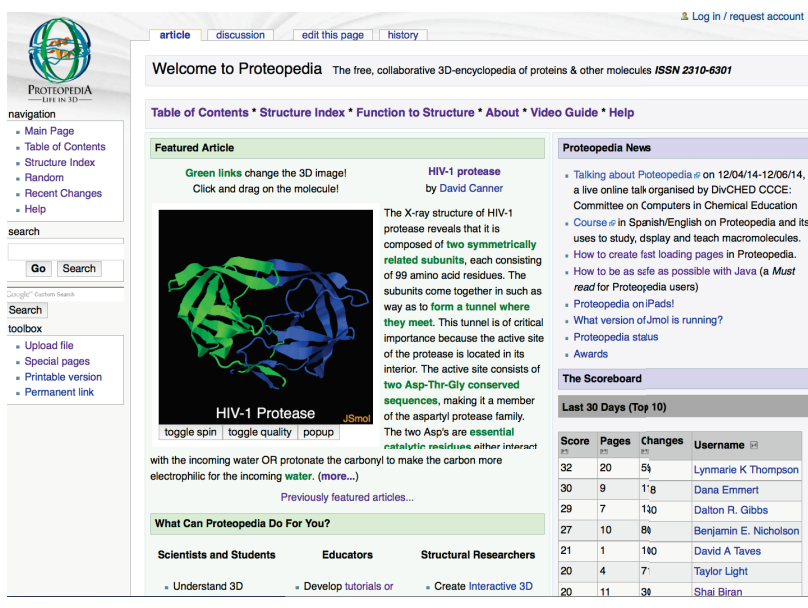

Figure 1. Proteopedia homepage: http://proteopedia.org

Keywords: Information Management, Wiki, education, structure/function 